\title{
CONGENITAL DEFORMITIES
}

\author{
T. J. S. Patterson, M.D., F.R.C.S. \\ Plastic Surgeon, The United Oxford Hospitals
}

\section{Incidence}

THERE is one anomaly of the upper limb in every 626 live births (Conway and Bowe, 1956).

\section{Etiology}

The association between thalidomide and severe malformations of the limbs has been clearly established (Smithells, 1962). In most other cases, questions to the parents of deformed children about incidents in pregnancy do not give any useful information about the cause of the deformity. The incidents which have impressed themselves on the parents' minds have usually occurred after the seventh week of pregnancy, by which time the hand is fully formed though in miniature (Bardeen and Lewis, 1901; Lewis, 1901).

Approximately $5 \%$ of hand deformities are inherited (Barsky, 1958). Either the deformity is transmitted in pure form through many generations, or a tendency to deformity of the limb is transmitted and the exact form varies (see also BirchJensen, 1949).

\section{Classification}

Many cases of congenital deformity of the hand present such a strange appearance that it may seem to be impossible to fit them into any sort of classification. Instead of studying severe malformations and trying to decide which is the more important part of a complex abnormality, it is easier to start with the less severe cases and to work peripherally to see the very mildest type of abnormality. Examples of very localized defects can be found which involve only one type of tissue, e.g. a skin web extending to the proximal interphalangeal joints, fusion of two carpal bones, or contraction of the capsule on the front of one interphalangeal joint.

It is then possible to trace each minimal abnormality through stages which become increasingly severe and widespread, both locally in the hand and spreading proximally up the limb. This method of study suggeste a classification which separates malformations of the hand into five main groups on a purely anatomical basis:

I. Webbing of fingers
(a) with normal digits; digits.

2. Abnormalities of position

(a) flexion contracture; (b) lateral displacement.

3. Absence of parts.

4. Ring-constrictions.

5. Excess tissue.

It is important not to push any scheme of classification too far since there will always be some overlap between the groups. A classification is, however, valuable if it enables a comparison to be made between different methods of treatment in similar cases. In practice this anatomical classification corresponds very well to a functional classification.

\section{General Considerations}

A child with a congenital deformity of the hand differs from an adult who loses part of his hand as the result of accident or disease. The child has never known the use of a normal hand and, until becoming selfconscious of his appearance, makes every effort to keep up with his fellows at home and at school. He develops trick movements to utilize every available scrap of tissue. Even in severe deformities, these patients achieve excellent function.

Deformities in other systems.-Limb deformities may be accompanied by multiple congenital anomalies which are incompatible with life. Other serious anomalies may influence the decision to undertake treatment, and may mean that the child can only be properly cared for in an institution. Defective intelligence prevents the child making full use of the limb and lessens his cooperation in treatment.

Abnormalities in the same limb.-In the same limb there may be deformities of the chest and shoulder-girdle. Absence of the pectoralis major is the commonest single congenital absence of muscle. It is not uncommonly associated with a deformity of the hand on the same side; the fingers are small and webbed-Poland's syndactyly (Clarkson, 1962). Absence of the pectoralis major causes very little disability. In girls there is usually underdevelopment of the breast on that side.

Shortening of the limb, as in failure of development of the humerus, causes difficulties with 
personal toilet which can sometimes be overcome by the suppleness of the back.

There may be radio-ulnar synostosis. If the shoulder is norrnal it will compensate for this, and the disability is slight.

Local condition in the hand.-Only in the mildest cases is the abnormality confined to one tissue. The worse the deformity, the greater are the changes in the surrounding tissues or in the whole limb. Joints may be stiff and muscles and tendons rudimentary; nerves and vessels are misplaced. These abnormalities may prejudice the results of major reconstructive surgery.

The timing of surgical treatment.-Early operative treatment has been recommended for two reasons. Firstly, because unless the deformed parts are freed and put into their correct positions, there will be secondary atrophy of muscles and stiffening of joints. It seems more likely, however, that these muscle and joint abnormalities are part of the original deformity. Secondly, since the child quickly develops abnormal movements to compensate for certain types of deformity, surgical treatment should be carried out before the pattern becomes firmly established. The child, is, however, quick to learn to use any new technique which becomes available to him as the result of surgery. In very young children the technical difficulties of operations and post-operative splinting are increased.

The timing in most cases will be governed by the school age of five. It is desirable that treatment shall be completed by that age and that the child's hand is then in the form that it will remain for the rest of his life.

The importance of sensation.-The importance of the hand as a sensory organ must always be borne in mind. Covering the hand with a prosthesis will interfere with this, as will reconstruction with skin flaps from a distance. If flap cover is required, the operation can often be planned to shift sensitive hand skin over the tactile areas, either by a local flap, or by 'filleting' a useless digit.

The place for prostheses.-Artificial arms are unsatisfactory compared with artificial legs. The urge to walk is so strong that an infant will make full use of an artificial leg and achieve excellent function (McKenzie, I957). An artificial arm is heavy and clumsy, and reduces the sensory area of the hand. The child can often only be persuaded with difficulty to keep it on, and great patience and ingenuity are required by parents and limb fitters. The prosthesis has to stand up to heavy wear and tear, and requires frequent replacement as the child grows. In spite of careful design, many patients with less severe deformities only wear their prostheses on social occasions; the cosmetic advantage of the prosthesis is more important than the functional. The appearance of the hand becomes increasingly important as the child grows older, and most adolescents will ask $\frac{\mathbb{Q}}{2}$ for some disguise for the deformity.

In some of the more severe deformities, however, a prosthesis may play an important part in treatment, and it is now felt that, in such cases, it shouldo be fitted early to coincide with normal motor development. A light, simple limb may be worn $\frac{\bar{\omega}}{\frac{5}{5}}$ from the age of 5 to 6 months to accustom the $\stackrel{\mathbb{Q}}{\square}$ child to it. Functional devices, such as the splithook, are introduced as the child becomes ready for them, so that he is efficient in the use of the prosthesis long before schooldays (James and $\vec{\omega}$ Lamb, 1963).

The increase in the number of severe deformi- 8 ties due to thalidomide has stimulated research 3 . into the powered prosthesis, such as the Heidelberg $\overrightarrow{0}$ limb. The development of these prostheses will lead to modifications in the type of surgical treatment which may be needed. The aim will be to retain or to provide some piece of tissue, how- $\mathrm{G}$ ever small, which can be made to act as a lever 음 controlling the switch of a powered mechanism. $\overrightarrow{-}$

The appearance of the hand.-In the treatment of deformities of the hand restoration of function takes first place. But the problem of the appearanee $\vec{\varnothing}$ of the hand becomes increasingly important as the child grows older. In reviewing a large number of these cases it has been surprising to find how widespread is the psychological disturbance in adolescence caused by the deformity, and what adverse effects this has on the use of the hand and $\bar{\varnothing}$ the response to treatment (Patterson, 1959). For this reason the appearance of a prosthesis is of the greatest importance. Girls will not wear a split- $\frac{}{3}$ hook, preferring the more natural looking 'dress' hand, in spite of the limitations on function which this imposes.

The reaction of a child to his deformity is largely determined by the attitude of his family. It is important that parents should be given advice $\delta$ at the earliest possible stage. They should be persuaded not to try to conceal the deformity and 0 not to do things for the child when he is clumsy or slow. It is important that he should not be o treated differently from other children. Normal independence is best developed by allowing him $ᄋ$ to make his own way among a large family or at $N$ school. Most of these children are quite capable $\underset{\mathrm{C}}{\mathrm{N}}$ of keeping up with their contemporaries at a normal 0 school, and it is most important that they should be enabled to do so.

\section{Webbing of Fingers (Syndactyly)}

This is probably the commonest single ab- 0 normality and is often found in combination with 
other malformations of the hand. It is important to separate cases of webbed fingers into two groups: $(a)$ in which the digits are otherwise normal; $(b)$ in which there are skeletal abnormalities.

(a) With normal digits.-This is often bilateral and familial, and the toes may also be affected. The middle and ring fingers are most commonly involved. In all cases a true web is rare. Usually the fingers are bound closely together by skin which may extend to the tips of the fingers. The principle of treatment is to make a skin-lined cleft which will not contract as the child grows. Operation is carried out when the child is about 4 years old. Occasionally a long finger may be distorted by being joined to a shorter finger, e.g. webbing between ring and little fingers; these should be separated earlier to prevent deformity of the longer finger.

When the fingers are separated there is a skin defect on the sides of the fingers which can rarely be made good by any form of local flap. A graft is required to prevent contracture. If the graft is laid into the defect in one piece the continuous marginal scar may contract. A small flap of skin at the base of the cleft should therefore be used to break the line of this scar.

(b) With abnormal digits.-Here the prognosis for function may depend more upon the skeletal abnormality than upon the webbing. The small webbed hand, which is the commonest type in this group, is usually unilateral and is not inherited. The toes are unaffected. Associated deformities are common, and the whole upper limb is usually smaller than the other side. The bones are malformed and there may be symphalangism with absence of interphalangeal joints. Each case should be assessed individually, and operation delayed until clinical and radiological evidence show that an individual digit is likely to be stable after separation. In many cases the separation of abnormal fingers is requested for cosmetic reasons. Division of the webs may not make the fingers any more useful, but the hand looks better, and it is possible to wear a glove and a wedding ring.

\section{Abnormalities of Position}

(a) Flexion contracture.-The normal infant holds his fingers and thumb flexed into the palm for the first few weeks of life. For this reason deformities such as flexion contracture and trigger thumb may not be noticed until some time after birth. In its simplest form the flexion contracture is seen as the 'congenital contracture of the little finger,' which is often bilateral and familial. There is contracture of all tissues, including the capsule, on the front of the proximal interphalangeal joint. All fingers may be involved in a similar process, and in the severest cases they are deviated to the ulnar side.

Trigger thumb is readily cured by incising the flexor sheath opposite the metacarpo-phalangeal joint as soon as the condition is recognized. The milder contractures of the fingers will be improved by passive stretching started at an early stage, accompanied by night splinting. Congenital contracture of the little finger is difficult to deal with. Release of the soft tissues has little effect, and it may be better to wait, until growth has ceased, before carrying out osteotomy of the proximal phalanx.

(b) Lateral displacement.-Minor displacements of one finger (clinodactyly) rarely cause interference with function.

Deviation at the wrist is commoner to the radial than to the ulnar side. The radial club hand is usually associated with absence of the radius, although deviation of the hand may occur when the radius is intact. The condition may be bilateral, and associated abnormalities are common; the hand and arm are small and the shouldergirdle is underdeveloped. The fingers may be thin and stiff, and the thumb and first metacarpal absent. One of the main disabilities is lack of power in the fingers. The greater the deviation at the wrist the more relaxed are the forearm muscles, and the less effective are the fingers in strength and range of movement. However, even when the thumb is missing the stiff fingers give an adequate grip for normal activities. The most powerful movement which these patients possess takes advantage of the fixed radial deviation of the hand. The limb is used as a hook. Straightening the wrist removes this powerful action.

The widespread changes in the limb make treatment difficult. Prolonged splinting from early life improves the position but adds little to the function. When the child starts to use the limb actively, the splint may become an encumbrance. In 1957 Entin and Petrie described their principles of treatment: splinting should be started early with light splints which interfere as little as possible with the use of the hand. If the hand cannot be straightened, the soft tissues on the radial side are lengthened. At the age of eight an autogenous graft of the proximal half of the fibula is wedged into the ulna to form a buttress for the carpus. Arthrodesis of the wrist is postponed until the hand is fully grown. (See also Pulvertaft and Reid, 1963.)
Absence of Parts
On the radial side of the hand the thumb may be unstable due to absence of the first metacarpal. The phalanges may be rudimentary and connected to the hand by a pedicle of varying thickness. In 
stabilizing such a thumb, the best that can be provided is a rigid post placed in a position to allow contact with the index finger. Even so, a child who has learnt an efficient 'pinch-grip' between the tips of the index and middle fingers, will not readily give this up to use a rigid post which may prove to be an encumbrance.

The thumb and first metacarpal may be completely absent. Abnormalities of the carpus on the radial side and absence of the radius and radial club-hand may be associated. The child with four normal fingers develops a very efficient grip between the tips of the index and middle fingers. If the thumb is absent on one side, pollicization of the index does not give a worth-while improvement in function. It is also important to remember that the four-finger hand looks natural; the absence of the thumb is not immediately apparent to a casual glance. In bilateral absence of the thumb, pollicization of the index may be carried out on one side. Bilateral absence of the thumb is, however, often associated with serious deformities of the rest of the limb, and the fingers may be stiff and unsuitable for transposition.

Digits may be absent on the ulnar side of the hand. This has little effect on function.

In the centre of the hand are the 'lobster-claw' deformities. Classically the central ray is missing, but it is commoner to find the central three rays deformed and the fingers reduced to blobs of soft tissue. Function in these cases may be very good. The central rudimentary digits are nearly always useless. X-rays will show whether it is possible to increase the span and allow larger objects to be grasped by deepening the cleft.

When the deformity is more severe, the radial and ulnar digits may also be rudimentary. If the digits are mobile, opposition may be possible after lengthening one of the digits. When there is no possibility of reconstructing an opposition mechanism, wedges of wood or cork may be made of such a size that they can be held in the rudimentary cleft; into these can be fixed tools, and knives and forks.

With more severe failures of development, excellent function may still be achieved. With one opposing digit on each hand, the child can keep up with his fellows at school and, in adult life, earn his living by work involving manual skill. These patients are successful because the digit which they possess is capable of opposing to the rest of the hand. With increasingly severe deformities there comes an abrupt change. With one opposable digit function may be nearly normal; if the digit is stiff, or flail, or cannot be made to oppose to any other part, function is grossly diminished. Occasionally a single short digit, for which it is not possible to build an opposition post can be incorporated in, and madis to work against, a prosthesis, or even to activat part of it.

Absence of all digits is comparatively commo and is generally unilateral. With one normal hand these children, who are characteristically highly intelligent, discover ways of carrying out moss? daily tasks. Their one complaint is that they have to have food cut up for them; this is a source of embarrassment at school. With the standar 'dress' hand, which is worn as a disguise for th 8 deformity, the child can achieve a very satisfactor grip for a knife or fork by wedging the implement. between the artificial fingers.

With more severe deformities the hand maz্ট articulate directly with the shoulder-girdle (phocos melus), or there may be complete absence of the limb. In these severe deformities the feet and. mouth become increasingly important. Childref with absence of the upper limbs, who have learnit to use their feet, will not persist with attempts tọ wear clumsy prostheses, but they may do so withy the newer powered appliances.

\section{Ring-Constrictions}

In this group are a number of varied condition which make up the ring-constriction syndrome (Patterson, 196I). These cases are frequenil associated with amniotic bands and it has been suggested that a band is the cause of the deformio ties. Streeter (1930) showed that it is more likets that both the deformities and the amniotic band are the result of the same developmental failureo A simple ring-constriction is probably a failure of development which affects primarily the sub $\overrightarrow{0}$ cutaneous tissue. As the ring becomes deeper? bone may be exposed, and other structures sucl as blood vessels are also affected. This is respon? sible for changes in distal parts such as lympho œdema. Digits may be webbed together but the web is always incomplete proximally. In the most severe cases the distal parts are amputated the bone protruding from the subcutaneous tissues like a guillotine amputation.

The shallower rings are treated for appearance only; they tend to become less obvious as the child grows. Double transposition flaps (Z-plasty are used to break the line of the circular scar this sometimes improves the odema of the distad part.

\section{Excess Tissue}

In this group there may be excessive number of parts or excessive size.

Polydactyly.-Some form of duplication of a digit is very common, ranging from a minute ta? to a fully formed digit or digits. The extra digit? 
which is often rudimentary, is usually marginally placed and commonly bilateral. In the severest forms there may be almost complete duplication of the limb.

The extra digit is usually easily removed. Difficulty sometimes arises in the case of the thumb where the two halves may be nearly symmetrical. Careful clinical and X-ray examination is required, accompanied sometimes by exploration under a tourniquet. As a general rule the more radially placed digit should be removed.

Excessive size (gigantism).-As distinct from hæmangiomatous or neurofibromatous gigantism, the enlargement in these congenital cases is due to a general overgrowth of all tissues. In addition to the increase in size of one or more fingers, there is also distortion of the fingers which becomes more marked with growth, so that in adult life the joints undergo early arthritic changes. Attempts to arrest growth when the optimum size has been reached have not been successful. Occasionally amputation of the terminal phalanx and removal of excess soft tissue may make a reasonable finger. Since many of them, however, become distorted and painful in adult life, amputation may be the only possible treatment.

\section{REFERENCES}

Bardeen, C. R., and Lewis, W. H. (1901): Development of the Limbs, Body-wall and Back in Man, Amer. F. Anat., r, I . BARSkY, A. J. (1958): 'Congenital Anomalies of the Hand and Their Surgical Treatment'. Springfield, Illinois: Charles C Thomas.

Birch-Jensen, A. (1949): 'Congenital Deformities of the Upper Extremities'. Opera ex Domo Biologiæ Hereditariæ Humanæ Universitatis Hafniensis', Vol. 19. Copenhagen.

Clarkson, P. (1962): Poland's Syndactyly, Guy's Hosp. Rep., III, 335.

Conway, H., and Bowe, J. (1956): Congenital Deformities of the Hands, Plast. reconstr. Surg., $18,286$.

Entin, M. A., and Petrie, J. G. (1957): Reconstruction of Congenital Absence of the Radius, Trans. Int. Soc. Plast. Surgs., p. 448 .

James, J. I. P., and Lamb, D. W. (r963): Congenital Abnormalities of the Limbs, Practitioner, r9r, I 59.

Lewis, W. H. (r 901 ): The Development of the Arm in Man, Amer. F. Anat., r, 145.

McKenzie, D. S. (1957): The Prosthetic Management of Congenital Deformities of the Extremities, $\mathcal{F}$. Bone $\mathcal{F}$. Surg., 39B, 233 .

Patterson, T. J. S. (1959): Congenital Deformities of the Hand, Ann. roy. Coll. Surg. Engl., $25,306$.

(1961): Congenital Ring-constrictions, Brit. F. plast. Surg., 14, 1 .

Pulvertaft, R. G., and Reid, D. A. C. (1963): Surgery of the Hand in Great Britain, Brit. Y. Surg., 50, 673.

Smithells, R. W. (1962): Thalidomide and Malformations in Liverpool, Lancet, i, 1270.

STreeter, G. L. (1930): Focal Deficiencies in Fetal Tissues and their Relation to Intra-uterine Amputation, Contr. Embryol. Carneg. Instn., 22, 1. 